\title{
THE NUMBER OF SOLUTIONS OF SOME CONGRUENCES MODULO A PRODUCT OF PRIMES( $\left.{ }^{1}\right)$ BY \\ L. CARLITZ AND A. L. WHITEMAN
}

TO PROFESSOR L. J. MORDELL ON HIS SEVENTY-FIFTH BIRTHDAY

1. Introduction. Let $p_{1}^{\alpha_{1}} p_{2}^{\alpha_{2}}, \cdots, p_{r}^{\alpha_{r}}$ be the prime factor decomposition of an odd integer $m$, and let $g_{i}(i=1, \cdots, r)$ be a primitive root of $p_{i}^{\alpha_{i}}$. By the Chinese Remainder Theorem the system of congruences

$$
g \equiv g_{1}\left(\bmod p_{1}^{\alpha_{1}}\right), \cdots, g \equiv g_{r}\left(\bmod p_{r}^{\alpha_{r}}\right)
$$

has a solution $g$ that is unique modulo $m$. The exponent $d$ of $g$ modulo $m$ is the least common multiple of $\phi\left(p_{1}^{\alpha_{1}}\right), \cdots, \phi\left(p_{r}^{\alpha_{r}}\right)$, where $\phi(n)$ is the Euler function. In this paper we consider the problem of determining the number $N(g)$ of solutions in $s, t$ of the congruence $g^{s}+1 \equiv g^{t}(\bmod m)$, where the values of $s$ and $t$ are each selected from the integers $0,1, \cdots, d-1$.

We discuss for the most part the case in which $m$ is the product of two distinct odd primes $p$ and $q$. If $e$ denotes the greatest common divisor of $p-1$ and $q-1$, then $d=(p-1)(q-1) / e$. Let $g$ denote a common primitive root of $p$ and $q$, and let $N(g)=N_{e}(g)$ denote the number of solutions in $s, t$ of the congruence $g^{s}+1 \equiv g^{t}(\bmod p q)$, where $0 \leqq s, t \leqq d-1$. In [3] the function $N_{e}(g)$ is required for the construction of certain types of residue difference sets. Indeed, it is this application of $N_{e}(g)$ that provided the motivation for the present paper (see the corollary of Theorem 3 in $\S 5$ ).

The case $e=2$ is treated in $\S 4$. We find that

$$
N_{2}(g)= \begin{cases}\frac{1}{4}(p q-2 p-2 q+5) & (p \neq \equiv q(\bmod 4)), \\ \frac{1}{4}(p q-2 p-2 q+7) & (p \equiv q \equiv 3(\bmod 4)) .\end{cases}
$$

Thus the expression for $N_{2}(g)$ is independent of the selection of $g$.

When $e>2$ the number $N_{e}(g)$ depends upon the particular choice of $g$. We show in $\S 3$ that the set $G$ of common primitive roots of $p$ and $q$ can be separated into $\phi(e)$ disjoint classes $G_{1}, \cdots, G_{\phi(e)}$ each containing $\phi(d)$ roots. The class $G_{i}$ has the following property: if $g_{i}$ is in $G_{i}$, then $G_{i}$ consists of the $\phi(d)$ numbers $g_{i}^{r}$ such that $1 \leqq r \leqq d-1$ and $(r, d)=1$. Furthermore, if $g_{i}$ is in $G_{i}$, then the value $N_{e}\left(g_{i}\right)$ depends only on the class $G_{i}$ and not on the choice of $g_{i}$ in $G_{i}$.

Received by the editors April 23, 1963.

(1) This research was partially supported by National Science Foundation grants G 16485 and G 24066. 
In the particular case $e=4$ the set $\bar{G}$ consists of two classes $G_{1}, G_{2}$. Let $g_{1} \in G_{1}$, $g_{2} \in G_{2}$. We prove in $\S 5$ that

$$
\begin{aligned}
& 4 N_{4}\left(g_{1}\right)=4 f f^{\prime}-f-f^{\prime}+\frac{3}{2}+\eta+\frac{1}{2}\left(1+2(-1)^{f+f^{\prime}}\right)(x X+y Y), \\
& 4 N_{4}\left(g_{2}\right)=4 f f^{\prime}-f-f^{\prime}+\frac{3}{2}+\eta+\frac{1}{2}\left(1+2(-1)^{f+f^{\prime}}\right)(x X-y Y),
\end{aligned}
$$

where $p=4 f+1=x^{2}+4 y^{2}$ and $q=4 f^{\prime}+1=X^{2}+4 Y^{2}$. The numbers $x, X$ are uniquely determined by the condition $x \equiv X \equiv 1(\bmod 4)$, whereas $y, Y$ are ambiguous. Moreover, the number $\eta$ is 0 or 1 according as $f f^{\prime}$ is even or odd. It follows that $N_{4}\left(g_{1}\right) \neq N_{4}\left(g_{2}\right)$. Essential use is made of this inequality in [3] where it is stated without proof as Lemma 6.

We also obtain corresponding results in the case $e=6$ (Theorem 4 in $\S 6$ ) and the case $e=8$ (Theorem 5 in $\S 7$ ). Finally, in $\S 8$ we discuss the case in which the modulus $m$ is the product of three distinct primes $p, q, r$. Under the assumption that $(p-1, q-1)=(q-1, r-1)=(r-1, p-1)=2$ we derive an explicit formula for $N(g)$ (Theorem 6).

The method consists in expressing $N(g)$ in terms of Jacobi sums. These sums are based on certain multiplicative characters defined in $\S 2$. Theorem 1 is the main tool.

2. The $\chi$ and $\psi$ characters. Let $p, q$ denote distinct odd primes. Let $g$ be a fixed primitive root of both $p$ and $q$, and let $e$ denote the greatest common divisor of $p-1$ and $q-1$. The exponent $d$ to which $g$ belongs modulo $p q$ is the least common multiple of $p-1$ and $q-1$. Consequently $d=(p-1)(q-1) / e$.

Let $\beta=\exp (2 \pi i / e)$ be a primitive eth root of unity. We define eth power characters $\chi(a)=\chi_{e}(a)$ modulo $p$ and $\psi(a)=\psi_{e}(a)$ modulo $q$ as follows: $\chi(a)=\beta^{u}$ if $a \equiv g^{u}(\bmod p)$ and $\chi(a)=0$ if $p \mid a ; \psi(a)=\beta^{v}$ if $a \equiv g^{v}(\bmod q)$ and $\psi(a)=0$ if $q \mid a$. It will be convenient to adopt the convention that $\chi^{n}(0)=0$ for $1 \leqq n \leqq e-1$ and $\chi^{n}(0)=1$ for $n=0$. We shall also let $\bar{\chi}(a)=\chi^{e-1}(a)$ denote the complex conjugate of $\chi(a)$.

We now prove the following criterion.

LEMMA 1 . Let s be a fixed integer such that $0 \leqq s \leqq d-1$ and $\left(g^{s}+1, p q\right)=1$. Then there corresponds an integer $t$ such that $0 \leqq t \leqq d-1$ and $g^{s}+1 \equiv g^{t}$ $(\bmod p q)$ if and only if $\chi\left(g^{s}+1\right)=\psi\left(g^{s}+1\right)$.

Proof. Consider the pair of congruences

$$
g^{s}+1 \equiv g^{u}(\bmod p), \quad g^{s}+1 \equiv g^{v}(\bmod q) .
$$

Then $g^{s}+1 \equiv g^{t}(\bmod p q)$ provided integers $h, k$ exist such that

$$
u+h(p-1)=v+k(q-1) \text {. }
$$

This will occur if and only if $u \equiv v(\bmod e)$. The assertion of the lemma follows at once. 
Let $T(g)$ denote the number of values of $s$ such that $0 \leqq s \leqq d-1$ and $\left(g^{s}+1, p q\right) \neq 1$. We next observe that the number $N(g)=N_{e}(g)$ of solutions $s, t$ of the congruence

$$
g^{s}+1 \equiv g^{t}(\bmod p q) \quad(0 \leqq s, t \leqq d-1)
$$

is related to the sum

$$
S(g)=\sum_{s=0}^{d-1} \sum_{n=0}^{e-1} \chi^{n}\left(g^{s}+1\right) \bar{\psi}^{n}\left(g^{s}+1\right)
$$

by means of the equation $S(g)=e N(g)+T(g)$. The reason is as follows. For each $s$ in (2.3) such that $\left(g^{s}+1, p q\right)=1$ the congruences in (2.1) imply that

$$
\sum_{n=0}^{e-1} \chi^{n}\left(g^{s}+1\right) \Psi^{n}\left(g^{s}+1\right)=\sum_{n=0}^{e-1} \beta^{(u-v) n}= \begin{cases}e & (u \equiv v(\bmod e)), \\ 0 & (u \neq v(\bmod e)) .\end{cases}
$$

On the other hand, for each $s$ in $(2.3)$ such that $\left(g^{s}+1, p q\right) \neq 1$, the inner sum in (2.3) has the value 1. It follows from Lemma 1 that the right member of (2.3) reduces to $e N_{e}(g)+T(g)$.

The problem of determining $N(g)$ has now been transformed into the problem of determining $S(g)$ and $T(g)$. An explicit formula for $T(g)$ is furnished by the following lemma.

LEMMA 2. Let $p-1=e f, q-1=e f^{\prime},\left(f, f^{\prime}\right)=1$. Then the number $T=T(g)$ of values of such that $0 \leqq s \leqq d-1$ and $\left(g^{s}+1, p q\right) \neq 1$ is given $b y$

$$
T= \begin{cases}f+f^{\prime} & \left(f f^{\prime} \text { even }\right), \\ f+f^{\prime}-1 & \left(f f^{\prime} \text { odd }\right)\end{cases}
$$

Proof. Clearly $f$ and $f^{\prime}$ cannot both be even. If $f f^{\prime}$ is odd, then $-1 \equiv g^{d / 2}(\bmod p q)$. But if $f f^{\prime}$ is even, then there is no value of $s$ such that $-1 \equiv g^{s}(\bmod p q)$, where $0 \leqq s \leqq d-1$. Put $N=N(s)=1+g^{s}$ and let $N_{p q}$ denote the number of values of $s(s=0,1, \cdots, d-1)$ for which $N$ is divisible by $p q$. Then $N_{p q}$ is equal to 0 or 1 according as $f f^{\prime}$ is even or odd. Also let $N_{p}$ denote the number of values of $s(s=0,1, \cdots, d-1)$ for which $N$ is divisible by $p$. As $s$ ranges from 0 to $d-1$, the least positive remainders of $g^{s}$ modulo $p$ range $f^{\prime}$ times over each of the integers between 1 and $p-1$. Hence $N_{p}=f^{\prime}$. Similarly if $N_{q}$ denotes the number of values of $s(s=0,1, \cdots, d-1)$ for which $N$ is divisible by $q$, then $N_{q}=f$. By a well-known combinatorial principle the number $T$ is equal to $N_{p}+N_{q}-N_{p q}$, which reduces to the right member of (2.4). This completes the proof of the lemma.

We turn to the problem of determining $S(g)$. Interchanging signs of summation in (2.3) we get $S(g)=\sum_{n=0}^{e-1} S_{n}$, where

$$
S_{n}=S_{n}(g)=\sum_{s=0}^{d-1} \chi^{n}\left(g^{s}+1\right) \Psi^{n}\left(g^{s}+1\right)
$$


Evidently $S_{n}=d$ for $n=0$ and $S_{n}=S_{e-n}$ for $1 \leqq n \leqq e-1$. For later applications we write $S(g)$ in the form

$$
S(g)=d+S_{e / 2}+\sum_{n=1}^{e / 2-1}\left(S_{n}+S_{n}\right)
$$

It should be noted that when $e=2$ the sum in (2.6) is vacuous.

The evaluation of $S_{n}$ is facilitated by expressing the sum in (2.5) terms of Jacobi sums depending on $\chi$ and $\psi$. For integers $m, n$ we define the Jacobi sum

$$
J_{\chi}(m, n)=\sum_{a+b \equiv 1(\bmod p)} \chi^{m}(a) \chi^{n}(b),
$$

the summation extending over all pairs of integers in the range $1 \leqq a, b \leqq p-1$ such that $a+b \equiv 1(\bmod p)$. We also define the corresponding Jacobi sum $J_{\psi}(m, n)$. Thus the sums $J_{\chi}(m, n), J_{\psi}(m, n)$ depend upon the choice of the common primitive root $g$ of the primes $p=e f+1, q=e f^{\prime}+1$.

The following well-known properties of $J_{\chi}(m, n)$ will be employed many times in the sequel.

(2.11) $J_{\chi}(m, n) J_{\chi}(-m,-n)=p$.

In (2.11) it is assumed that no one of $m, n, m+n$ is divisible by $e$.

The Jacobi sum $J_{\chi}(m, n)$ is closely related to the Lagrange sum defined by

$$
\tau(\alpha)=\sum_{a=1}^{p-1} \alpha^{\text {ind } a y a} \quad\left(g^{\text {ind } a} \equiv a(\bmod p)\right),
$$

where $\alpha$ denotes a root of the equation $\alpha^{p-1}=1$, and $\zeta=\exp (2 \pi i / p)$. Indeed we have the formula

$$
J_{\chi}(m, n)=\tau\left(\beta^{m}\right) \tau\left(\beta^{n}\right) / \tau\left(\beta^{m+n}\right)
$$

when $m+n$ is not divisible by $e$. A deeper property of (2.12) is given by the formula

$$
\tau(-1) \tau\left(\alpha^{2}\right)=\alpha^{2 m} \tau(\alpha) \tau(-\alpha),
$$

where the integer $m$ is defined by the congruence $g^{m} \equiv 2(\bmod p)$.

Formulas $(2.8), \cdots,(2.14)$ are developed, for example, in an important paper of Dickson [1] on the theory of cyclotomy. The notation $J_{\chi}(m, n)$ in this paper corresponds to Dickson's $R(m, n)$.

We shall require the following two lemmas. 
LEMMA 3. If $a$ is an integer not divisible by $p$, and $n$ is an arbitrary integer, then

$$
\sum_{u=1}^{p-1} \chi^{n}\left(a u^{e}+1\right)=\sum_{m=0}^{e-1} \chi^{m}(-1) \bar{\chi}^{m}(a) J_{\chi}(m, n) .
$$

Proof. In the left member of (2.15) the number $u^{e}$ runs $e$ times over the $f$ incongruent $e$ th power residues of $p$. Since the sum $1+\chi(r)+\cdots+\chi^{e-1}(r)$ equals $e$ or 0 according as $r$ is an $e$ th power residue or not, we find that

$$
\begin{aligned}
\sum_{u=1}^{p-1} \chi^{n}\left(a u^{e}+1\right) & =\sum_{u=1}^{p-1} \sum_{m=0}^{e-1} \bar{\chi}^{m}(a) \chi^{m}(a u) \chi^{n}(a u+1) \\
& =\sum_{m=0}^{c-1} \bar{\chi}^{m}(a) \sum_{u=1}^{p-1} \chi^{m}(u) \chi^{n}(u+1) .
\end{aligned}
$$

When $u$ is replaced by $-u$ the inner sum becomes $\chi^{m}(-1) J_{\chi}(m, n)$. This completes the proof of Lemma 3.

If $a \equiv g^{k}(\bmod p)$, then $\chi(a)=\beta^{k}$; moreover, $\chi(-1)=\beta^{e f / 2}=(-1)^{f}$. Hence (2.15) may also be written in the form

$$
\sum_{u=1}^{p-1} \chi^{n}\left(g^{k} u^{e}+1\right)=\sum_{m=0}^{e-1}(-1)^{m f} J_{\chi}(m, n) \beta^{-m k} .
$$

LEMMA 4. Let $p-1=e f, q-1=e f^{\prime},\left(f, f^{\prime}\right)=1$. Let $S_{n}(g)$ be the sum defined in (2.5). Then

$$
S_{n}(g)=\frac{1}{e} \sum_{s=0}^{e-1}(-1)^{s\left(f+f^{\prime}\right)} J_{\chi}(s, n) J_{\bar{\psi}}(s, n) .
$$

Proof. To evaluate the right member of (2.5) we put

$$
s=k+(q-1) r+(p-1) t \quad\left(0 \leqq k<e ; 0 \leqq r<f, 0 \leqq t<f^{\prime}\right) .
$$

Since $d=e f f^{\prime}$ there are $d$ values of $s$ in (2.18). No two of these values of $s$ are in the same residue class modulo $d$. Hence we find that

$$
\begin{aligned}
S_{n}(g) & =\sum_{k=0}^{e-1} \sum_{r=0}^{f-1} \sum_{t=0}^{f^{\prime}-1} \chi^{n}\left(g^{k+(q-1) r}+1\right) \psi^{n}\left(g^{k+(p-1) t}+1\right) \\
& =\frac{1}{e^{2}} \sum_{k=0}^{e-1} \sum_{u=1}^{p-1} \chi^{n}\left(g^{k} u^{e}+1\right) \sum_{v=1}^{q-1} \psi^{n}\left(g^{k} v^{e}+1\right) .
\end{aligned}
$$

Substituting from (2.16) and changing the order of summation we get

$$
S_{n}(g)=\frac{1}{e^{2}} \sum_{s, t=0}^{e-1}(-1)^{s f+t f^{\prime}} J_{\chi}(s, n) J_{\bar{\psi}}(t, n) \sum_{k=0}^{e-1} \beta^{-\dot{k}(s-t)}
$$

The inner sum vanishes unless $s=t$. The identity in (2.17) is an immediate consequence. 
In order to summarize the results of this section we find it convenient to put

$$
M=\frac{(p-2)(q-2)-1}{e}
$$

Then we can write

$$
M+\eta=d-T,
$$

where $T$ is given in (2.4), and $\eta=0$ or 1 according as $f f^{\prime}$ is even or odd. From the line immediately following (2.3) we have the equation $S(g)=e N(g)+T(g)$. The following theorem now follows from (2.6) and (2.20).

THEOREM 1. Let $S_{n}(g)$ be the sum in (2.17). Then the number $N_{e}(g)$ of solutions in $s, t(0 \leqq s, t \leqq d-1)$ of the congruence $g^{s}+1 \equiv g^{t}(\bmod p q)$ is furnished by the formula

$$
e N_{e}(g)=M+\eta+S_{e / 2}(g)+\sum_{n=1}^{e / 2-1}\left(S_{n}(g)+S_{n}(g)\right),
$$

where the sum is vacuous for $e=2$.

3. The common primitive roots of $p$ and $q$. The value $N_{e}(g)$ depends in general on the choice of the common primitive root $g$ of $p$ and $q$. In this section we shall establish three lemmas giving information about the set of such common primitive roots.

LEMMA 5. Let $p$ and $q$ denote two distinct odd primes; let e denote the greatest common divisor of $p-1$ and $q-1$, and put $(p-1)(q-1)=d e$. Then the number $n_{e}(p, q)$ of common primitive roots of $p$ and $q$ in a reduced residue system modulo $p q$ is given by

$$
n_{e}(p, q)=\phi(e) \phi(d)
$$

where $\phi(m)$ is Euler's function.

Proof. By the Chinese Remainder Theorem the number $n_{e}(p, q)$ is equal to $\phi(p-1) \phi(q-1)$. We now apply the well-known formula

$$
\phi(m n)=\frac{P \phi(m) \phi(n)}{\phi(P)}
$$

where $P$ is the product of the primes common to $m$ and $n$. Put $p-1=e f$, $q-1=e f^{\prime},\left(f, f^{\prime}\right)=1$. Then the product $P$ of primes common to $e$ and $f$ is also the product of primes common to $e f^{\prime}$ and $f$. By (3.2) we get

$$
\phi(p-1)=\frac{P \phi(e) \phi(f)}{\phi(P)}, \quad \phi(d)=\frac{P \phi(q-1) \phi(f)}{\phi(P)} .
$$


Hence the product $\phi(p-1) \phi(q-1)$ reduces to the right member of (3.1). This proves the lemma.

Let $\bar{G}$ denote the set of common primitive roots of $p$ and $q$. If $g$ is in $\bar{G}$, then $g$ has the exponent $d$ modulo $p q$. Furthermore, the number $g^{r}$ is in $\bar{G}$ if and only if $(r, d)=1$. When $(r, d)=1$ the set of powers of $g$ modulo $p q$ is the same as the set of powers of $g^{r}$. In view of Lemma 5 the following conclusion may now be asserted.

LEMMA 6 . The $\phi(e) \phi(d)$ roots in $\bar{G}$ can be separated into $\phi(e)$ disjoint classes $G_{1}, G_{2}, \cdots, G_{\phi(e)}$, each containing $\phi(d)$ roots. These classes can be characterized as follows: If $g_{i}$ is in $G_{i}$, then $G_{i}$ consists of the $\phi(d)$ numbers

$$
g_{i}^{r} \quad(r=1, \cdots, d-1 ;(r, d)=1) .
$$

In the rest of this section we assume that $e=4$ or 6 so that $\phi(e)=2$. Then by Lemma 6 the set $\bar{G}$ of common primitive roots of $p$ and $q$ modulo $p q$ can be separated into two classes $G, G^{\prime}$. If $g \in G, g^{\prime} \in G^{\prime}$, then every root in $G$ is a power of $g$, while every root in $G^{\prime}$ is a power of $g^{\prime}$. We now fix $g, g^{\prime}$ and put

$$
g \equiv g^{\prime r}(\bmod p), \quad g \equiv g^{\prime s}(\bmod q) .
$$

The exponents $r, s$ in (3.3) are such that $(r, p-1)=(s, q-1)=1$; in particular, $(r, e)=(s, e)=1$. Since $e=4$ or 6 it follows that $r \equiv \pm 1(\bmod e), s \equiv \pm 1(\bmod e)$. We next show that

$$
r \not \equiv s \quad(\bmod e)
$$

Otherwise, since $(p-1, q-1)=e$, the congruence $r \equiv s(\bmod e)$ implies the existence of integers $h, k$ such that

$$
r+h(p-1)=s+k(q-1) .
$$

Putting $t=r+h(p-1)$ we deduce from (3.3) and (3.5) that $g \equiv g^{\prime t}(\bmod p q)$, so that $g$ is a power of $g^{\prime}$ in violation of the definition of the classes $G, G^{\prime}$. As a consequence of (3.4) we have the following useful lemma.

LEMMA 7. Let $e=4$ or 6 and let $g \in G, g^{\prime} \in G^{\prime}$ be fixed primitive roots of both $p$ and $q$. Let $a$ be an integer relatively prime to $p$ and $q$. Then the corresponding characters $\chi(a), \psi(a)$ and $\chi^{\prime}(a), \psi^{\prime}(a)$ are so related that one of the following two cases holds:

(i) $\chi(a)=\chi^{\prime}(a)$ and $\psi(a)=\bar{\psi}^{\prime}(a)$,

(ii) $\chi(a)=\bar{\chi}^{\prime}(a)$ and $\psi(a)=\psi^{\prime}(a)$.

Proof. Consider the pair of congruences

$$
a \equiv g^{u} \quad(\bmod p), \quad a \equiv g^{v} \quad(\bmod q),
$$

so that $\chi(a)=\beta^{u}, \psi(a)=\beta^{v}$. Let $\chi^{\prime}(a), \psi^{\prime}(a)$ be the characters constructed 
using $g^{\prime}$ in place of $g$. Then the congruences in (3.3) and (3.6) imply that $\chi^{\prime}(a)=\beta^{r u}$, $\psi^{\prime}(a)=\beta^{s v}$. Applying (3.4) we obtain case (i) or case (ii) of the lemma according as $r \equiv \pm 1(\bmod e)$. This completes the proof.

Lemma 7 will be used in $\S \S 5$ and 6 to distinguish the value of $N_{e}(g)$ from $N_{e}\left(g^{\prime}\right)$.

4. The case $e=2$. We now apply the results of $\$ 2$ to evaluate $N_{2}(g)$. Let $p-1=2 f, q-1=2 f^{\prime},\left(f, f^{\prime}\right)=1$. Furthermore, put $M=((p-2)(q-2)-1) / 2$. By Theorem 1 with $e=2$ we have $2 N_{2}(g)=M+\eta+S_{1}(g)$, where

$$
2 S_{1}(g)=J_{x}(0,1) J_{\bar{\psi}}(0,1)+(-1)^{f+f^{\prime}} J_{x}(1,1) J_{\bar{\psi}}(1,1) .
$$

Since $\beta=-1$ when $e=2$ the character $\chi(a)$ modulo $p$ reduces to the Legendre symbol $(a \mid p)$. The Jacobi sum in (2.7) becomes in turn

$$
J_{x}(m, n)=\sum_{a=1}^{p-1}\left(\frac{a}{p}\right)^{m}\left(\frac{1-a}{p}\right)^{n} .
$$

Clearly $J_{x}(0,1)=-1$. Moreover

$$
J_{\chi}(1,1)=\sum_{a=1}^{p-1}\left(\frac{a}{p}\right)\left(\frac{1-a}{p}\right)=\sum_{a=1}^{p-1}\left(\frac{\bar{a}-1}{p}\right)=-\left(\frac{-1}{p}\right)=-(-1)^{f},
$$

where $\bar{a}$ has been chosen so that $a \bar{a} \equiv 1(\bmod p)$. Substituting these values into (4.1) we get $S_{1}(g)=1$. The results of this section may now be summarized in the following theorem.

THEOREM 2. When $e=2$ the number of solutions of (2.2) is equal to

$$
N_{2}(g)= \begin{cases}\frac{1}{4}(p q-2 p-2 q+5) & \left(f f^{\prime} \text { even }\right), \\ \frac{1}{4}(p q-2 p-2 q+7) & \left(f f^{\prime} \text { odd }\right) .\end{cases}
$$

It is evident from (4.2) that the value of $N_{2}(g)$ does not depend upon the particular choice of $g$.

5. The case $e=4$. Let $p-1=4 f, q-1=4 f^{\prime},\left(f, f^{\prime}\right)=1$, and let $g$ be a common primitive root of $p$ and $q$. We proceed to evaluate $N_{4}(g)$ by means of the machinery developed in $\S 2$. In view of Theorem 1 we must first evaluate $S_{1}(g)$ and $S_{2}(g)$.

By $(2.8)$ and $(2.10)$ we have $J_{x}(0,1)=-1, J_{x}(2,1)=(-1)^{f} J_{x}(1,1)$ and $J_{x}(3,1)=-(-1)^{f}$. Hence (2.17) with $e=4, n=1$ becomes

$$
S_{1}(g)=\frac{1}{2}+\frac{1}{2}(-1)^{f+f^{\prime}} J_{x}(1,1) J_{\bar{\psi}}(1,1) .
$$

Again, from (2.8), (2.9) and (2.10) we get $J_{\chi}(0,2)=J_{\chi}(2,2)=-1$, 
$J_{\chi}(1,2)=(-1)^{f} J_{\chi}(1,1)$ and $J_{\chi}(3,2)=(-1)^{f} J_{\bar{\chi}}(1,1)$. Consequently (2.17) with $e=4, n=2$ reduces to

$$
S_{2}(g)=\frac{1}{2}+\frac{1}{4}\left(J_{\chi}(1,1) J_{\bar{\psi}}(1,1)+J_{\bar{\chi}}(1,1) J_{\psi}(1,1)\right) .
$$

For simplicity we write $J_{\chi}=J_{\chi}(1,1), J_{\psi}=J_{\psi}(1,1)$. Then Theorem 1 with $e=4$ in conjunction with (5.1) and (5.2) yields

$$
4 N_{4}(g)=M+\eta+\frac{3}{2}+\frac{1}{4}(1+2 \chi(-1) \psi(-1))\left(J_{\chi} J_{\bar{\psi}}+J_{\bar{\chi}} J_{\psi}\right)
$$

where $M=((p-2)(q-2)-1) / 4$. The value of $N_{4}(g)$ furnished by (5.3) depends upon the particular choice of $g$. The nature of this dependence is revealed by Lemma 7. In the sense of this lemma let $g \in G, g^{\prime} \in G^{\prime}$. Then the value of $N_{4}\left(g^{\prime}\right)$ is given by

$$
4 N_{4}\left(g^{\prime}\right)=M+\eta+\frac{3}{2}+\frac{1}{4}(1+2 \chi(-1) \psi(-1))\left(J_{\chi} J_{\psi}+J_{\bar{\chi}} J_{\bar{\psi}}\right)
$$

Following Dickson (compare $[1,(50)])$ we now put

$$
J_{\chi}(1,1)=-x+2 \beta y, \quad J_{\psi}(1,1)=-X+2 \beta Y,
$$

where $\beta^{2}=-1$. From (2.11) it follows that

$$
p=x^{2}+4 y^{2}, \quad q=X^{2}+4 Y^{2} \text {. }
$$

Dickson [1, pp. 400-401] proved that the signs of $x$ and $X$ in (5.6) are uniquely determined by the conditions $x \equiv X \equiv 1(\bmod 4)$; however, $y$ and $Y$ are twovalued, depending on the choice of the primitive root $g$. Substituting from (5.5). into (5.3) and (5.4) we obtain

THEOREM 3. When $e=4$ the number of solutions of (2.2) satisfies the formulas

$$
\begin{aligned}
& 4 N_{4}(g)=M+\eta+\frac{3}{2}+\frac{1}{2}\left(1+2(-1)^{f+f^{\prime}}\right)(x X+4 y Y), \\
& 4 N_{4}\left(g^{\prime}\right)=M+\eta+\frac{3}{2}+\frac{1}{2}\left(1+2(-1)^{f+f^{\prime}}\right)(x X-4 y Y) .
\end{aligned}
$$

The following corollary of Theorem 3, stated without proof in [3, Lemma 6], plays a central role in the construction of a special family of difference sets [3, Theorem 4].

Corollary. If $N_{+}(g), N_{4}\left(g^{\prime}\right)$ correspond to $g \in G, g^{\prime} \in G^{\prime}$ respectively, then the following inequality holds:

$$
N_{4}(g) \neq N_{4}\left(g^{\prime}\right)
$$


Proof. Subtracting (5.8) from (5.7) we get

$$
N_{4}(g)-N_{4}\left(g^{\prime}\right)=\left(1+2(-1)^{f+f^{\prime}}\right) y Y .
$$

The inequality (5.9) follows at once.

Theorem 3 has the following interpretation. By a well-known theorem [2, p. 128] there are exactly two representations of $p q$ in the form $a^{2}+4 b^{2}$ with $a \equiv 1(\bmod 4)$ and $b$ indeterminate. Let

$$
p q=a^{2}+4 b^{2}, \quad p q=a^{\prime 2}+4 b^{\prime 2} \quad\left(a \equiv a^{\prime} \equiv 1(\bmod 4)\right)
$$

denote these two representations. From (5.6) we have

$$
p q=(x X \pm 4 y Y)^{2}+4(x Y \mp y X)^{2} .
$$

Hence formula (5.7) may be written alternatively in the form

$$
16 N_{4}(g)= \begin{cases}p q-2 p-2 q+9-2 a & \left(f f^{\prime} \text { even }\right) \\ p q-2 p-2 g+13+6 a & \left(f f^{\prime} \text { odd }\right)\end{cases}
$$

where $a$ appears in one of the two decompositions in (5.10), say the first. In view of the corollary the corresponding formula for $N_{4}\left(g^{\prime}\right)$ is obtained from (5.11) by replacing $g$ by $g^{\prime}$ and $a$ by $a^{\prime}$.

6. The case $e=6$. Throughout this section let $p-1=6 f, q-1=6 f^{\prime}$, $\left(f, f^{\prime}\right)=1$. The number $\beta$ is thus a primitive sixth root of unity so that $\beta^{2}=\beta-1$. We shall evaluate $N_{6}(g)$, where $g$ is a primitive root of both $p$ and $q$. In order to apply Theorem 1 we first obtain expressions for $S_{1}(g), S_{2}(g), S_{3}(g)$ in terms of Jacobi sums.

In (2.4) replace $\alpha$ by $\beta$ and then multiply both members of the resulting equation by $\tau\left(\beta^{2}\right) / \tau\left(\beta^{3}\right) \tau\left(\beta^{4}\right)$. By (2.10) and (2.13) we get

$$
J_{\chi}(4,1)=\chi^{4}(2) J_{\chi}(2,1), \quad J_{\chi}(2,2)=\chi^{2}(2) J_{\chi}(2,1),
$$

where $\chi(2)=\beta^{m}$ and $g^{m} \equiv 2(\bmod p)$. For brevity we shall write $J_{\chi}$ in place of $J_{\chi}(2,1)$. Using $(2.8),(2.9),(2.10)$ in connection with (6.1) we may express each of the eighteen sums $J_{\chi}(s, n)(0 \leqq s \leqq 5,1 \leqq n \leqq 2)$ in terms of $J_{\chi}$. The formulas are given in the following table.

\begin{tabular}{cccc}
\hline & $n=1$ & $n=2$ & $n=3$ \\
\hline$J_{\chi}(0, n)$ & -1 & -1 & -1 \\
$J_{\chi}(1, n)$ & $\chi(-1) \chi^{4}(2) J_{\chi}$ & $J_{\chi}$ & $\chi(-1) J_{\chi}$ \\
$J_{\chi}(2, n)$ & $J_{\chi}$ & $\chi^{2}(2) J_{\chi}$ & $J_{\chi}$ \\
$J_{\chi}(3, n)$ & $\chi(-1) J_{\chi}$ & $J_{\chi}$ & $-\chi(-1)$ \\
$J_{\chi}(4, n)$ & $\chi^{4}(2) J_{\chi}$ & -1 & $J_{\bar{\chi}}$ \\
$J_{\chi}(5, n)$ & $-\chi(-1)$ & $\chi^{2}(2) J_{\bar{\chi}}$ & $\chi(-1) J_{\bar{\chi}}$ \\
\hline
\end{tabular}


We employ Lemma 4 with $e=6$. Substituting the results in the table into (2.17) we get for $n=1,2,3$

$$
\begin{aligned}
S_{1}(g)= & \frac{1}{3}\left(1+J_{\chi} J_{\bar{\psi}}+\chi^{4}(2) \psi^{2}(2) J_{\chi} J_{\bar{\psi}}\right) . \\
S_{2}(g)= & \frac{1}{6}\left(2+2_{\chi}(-1) \psi(-1) J_{\chi} J_{\bar{\psi}}+\chi^{2}(2) \psi^{4}(2) J_{\chi} J_{\bar{\psi}}\right. \\
& \left.+\chi(-1) \psi(-1) \chi^{2}(2) \psi^{4}(2) J_{\bar{\chi}} J_{\psi}\right) . \\
S_{3}(g)= & \frac{1}{3}\left(1+J_{\chi} J_{\bar{\psi}}+J_{\bar{\chi}} J_{\psi}\right) .
\end{aligned}
$$

We are now in the position to evaluate $N_{6}(g)$ by means of Theorem 1 with $e=6$. Noting that $\chi(-1) \psi(-1)$ is equal to \pm 1 according as $f f^{\prime}$ is odd or even, and using (6.2), (6.3), (6.4) we get

$$
36 N_{6}(g)=6 M+6 \eta+10+\delta J_{\chi} J_{\bar{\psi}}+\delta J_{\bar{\chi}} J_{\psi},
$$

where $M=((p-2)(q-2)-1) / 6$, and where

$$
\delta= \begin{cases}2+\chi^{2}(2) \psi^{4}(2)+\chi^{4}(2) \psi^{2}(2) & \left(f f^{\prime} \text { even }\right), \\ 6+\chi^{2}(2) \psi^{4}(2)+3 \chi^{4}(2) \psi^{2}(2) & \left(f f^{\prime} \text { odd }\right) .\end{cases}
$$

The expression for $N_{6}(g)$ in (6.5) depends upon the choice of $g$. Let $g \in G$, $g^{\prime} \in G^{\prime}$ in the sense of Lemma 7. It follows from this lemma that

$$
36 N_{6}\left(g^{\prime}\right)=6 M+6 \eta+10+\delta^{\prime} J_{\chi} J_{\psi}+\bar{\delta}^{\prime} J_{\bar{\chi}} J_{\bar{\psi}},
$$

where

$$
\delta^{\prime}=\left\{\begin{array}{ll}
2+\chi^{2}(2) \psi^{2}(2)+\chi^{4}(2) \psi^{4}(2) & \left(f f^{\prime} \text { even }\right), \\
6+\chi^{2}(2) \psi^{2}(2)+3 \chi^{4}(2) \psi^{4}(2) & \left(f f^{\prime} \text { odd }\right) .
\end{array} .\right.
$$

When $f f^{\prime}$ is even it is clear from (6.6), (6.6) that $\delta=\bar{\delta}$ and $\delta^{\prime}=\bar{\delta}^{\prime}$. More precisely, $\delta=4$ or 1 according as $\chi^{2}(2) \psi^{4}(2)=1$ or not, and $\delta^{\prime}=4$ or 1 according as $\chi^{2}(2) \psi^{2}(2)=1$ or not. When $f^{\prime}$ is odd, $\delta=10,5-2 \beta, 3+2 \beta$ according as $\chi^{2}(2) \psi^{4}(2)=1, \beta^{2}, \beta^{4}$ respectively, and $\delta^{\prime}=10,5-2 \beta, 3+2 \beta$ according as $\chi^{2}(2) \psi^{2}(2)=1, \beta^{2}, \beta^{4}$ respectively. To simplify further we consider separately the cases (i) $f f^{\prime}$ even, $\delta=1$, (ii) $f f^{\prime}$ even, $\delta=4$, (iii) $f f^{\prime}$ odd, $\delta=10$, (iv) $f f^{\prime}$ odd, $\delta=5-2 \beta$, (v) $f f^{\prime}$ odd, $\delta=3+2 \beta$; (i)' $f f^{\prime}$ even, $\delta^{\prime}=1$, (ii) $f f^{\prime}$ even, $\delta^{\prime}=4$, (iii)' $f f^{\prime}$ odd, $\delta^{\prime}=10$, (iv) $f f^{\prime}$ odd, $\delta^{\prime}=5-2 \beta$, (v) $f f^{\prime}$ odd, $\delta^{\prime}=3+2 \beta$.

Following Dickson $[1,(85)]$ we put

$$
J_{\chi}(2,1)=-a+b(2 \beta-1), \quad J_{\psi}(2,1)=-A+B(2 \beta-1),
$$

where, in view of (2.11), we have

$$
p=a^{2}+3 b^{2}, \quad q=A^{2}+3 B^{2} .
$$


Dickson $[1$, p. 409$]$, proved that $a \equiv 1$ or $4(\bmod 6)$ according as $f$ is even or odd; similarly, $A \equiv 1$ or $4(\bmod 6)$ according as $f^{\prime}$ is even or odd. On the other hand, $b$ and $B$ are uniquely determined by (6.8) except for sign. Substituting from (6.7) into (6.5) and (6.5)' we obtain the following theorem.

THEOREM 4. When $e=6$ the number of solutions of (2.2) satisfies the formulas

$$
\begin{aligned}
& 36 N_{6}(g)= \begin{cases}6 M+10+2 \delta(a A+3 b B) & (\text { cases } \mathrm{i}, \mathrm{ii}), \\
6 M+16+20(a A+3 b B) & (\text { case iii), } \\
6 M+16+8(a A+3 b B) \pm 6(a B-b A) & (\text { cases iv, }),\end{cases} \\
& 36 N_{6}\left(g^{\prime}\right)= \begin{cases}6 M+10+2 \delta^{\prime}(a A-3 b B) & (\text { cases i', ii'), } \\
6 M+16+20(a A-3 b B) & (\text { case iii'), } \\
6 M+16+8(a A-3 b B) \pm 6(a B+b A) & \left(\text { case iv', } \mathrm{v}^{\prime}\right) .\end{cases}
\end{aligned}
$$

The ambiguous sign is plus in cases iv, $\mathrm{v}^{\prime}$ and minus in cases $\mathrm{v}, \mathrm{iv}^{\prime}$.

In view of the corollary to Theorem 3 it is natural to inquire if the value $N_{6}(g)$ necessarily differs from $N_{6}\left(g^{\prime}\right)$. Actually, Theorem 4 implies in some instances but not in general that $N_{6}(g) \neq N_{6}\left(g^{\prime}\right)$. Thus if $f f^{\prime}$ is even and $\delta=\delta^{\prime}$, then $36 N_{6}(g)-36 N_{6}\left(g^{\prime}\right)=12 \delta b B$. Again if $f f^{\prime}$ is odd and $\delta=\delta^{\prime}=10$, then $36 N_{6}(g)-36 N_{6}\left(g^{\prime}\right)=120 b B$. On the other hand, consider the example $p=7$, $q=19, g=3, g^{\prime}=10$, in which $\chi(2)=\chi^{\prime}(2)=\beta^{2}, \psi(2)=\bar{\psi}^{\prime}(2)=\beta$. Then $J_{\chi}=1+2 \beta, J_{\psi}=-3-2 \beta$ so that $a=-2, b=1, A=4, B=-1$. Moreover, $f f^{\prime}$ is odd, $\delta=5-2 \beta, \delta^{\prime}=10$. We deduce from case iv of (6.9) and case iii' of (6.9)' that $N_{6}(g)=N_{6}\left(g^{\prime}\right)=0$.

From (6.8) we obtain $p q=(a A \pm 3 b B)^{2}+3(a B \mp b A)^{2}$. Hence (6.9) may be recast in the following form:

$$
36 N_{6}(g)= \begin{cases}p q-2 p-2 q+13+2 \delta c & (\text { cases i, ii) } \\ p q-2 p-2 q+19+20 c & \text { (case iii) } \\ p q-2 p-2 q+19+8 c \pm 6 d & \text { (cases iv, v) }\end{cases}
$$

where $c^{2}+3 d^{2}$ is one of the two representations of $p q$ as a square plus three times a square. The sign of $c$ is uniquely determined by the condition that $c \equiv 4$ or $1(\bmod 6)$ according as $f f^{\prime}$ is even or odd. On the other hand, $d$ is ambiguously determined.

7. The case $e=8$. In this section we shall obtain expressions for $N_{8}(g)$, where $g$ is a common primitive root of $p=8 f+1, q=8 f^{\prime}+1$ and $\left(f, f^{\prime}\right)=1$. In order to apply Theorem 1 with $e=8$ we first derive expressions for $S_{1}(g)$, $S_{2}(g), S_{3}(g), S_{4}(g)$.

Let $\beta=\exp (2 \pi i / 8)$; then $\chi(2)=\beta^{m}$ where $g^{m} \equiv 2(\bmod p)$. It turns out that each of the thirty-two Jacobi sums $J_{\chi}(s, n)(0 \leqq s \leqq 7,1 \leqq n \leqq 4)$ may be expressed in terms of either $J_{\chi}(2,2)$ or $J_{\chi}(3,1)$. The results are given compactly in the following table. 


\begin{tabular}{|c|c|c|}
\hline & $n=1$ & $n=2$ \\
\hline$J_{\chi}(0, n)$ & -1 & -1 \\
\hline$J_{\chi}(1, n)$ & $\chi(-1) \chi^{6}(2) J_{\chi}(3,1)$ & $\chi(-1) \chi^{6}(2) J_{\chi}(2,2)$ \\
\hline$J_{\chi}(2, n)$ & $\chi(-1) \chi^{6}(2) J_{\chi}(2,2)$ & $J_{\chi}(2,2)$ \\
\hline$J_{\chi}(3, n)$ & $J_{\chi}(3,1)$ & $\chi^{2}(2) J_{\chi}(3,1)$ \\
\hline$J_{\chi}(4, n)$ & $\chi(-1) J_{\chi}(3,1)$ & $J_{\chi}(2,2)$ \\
\hline$J_{\chi}(5, n)$ & $\chi^{6}(2) J_{\chi}(2,2)$ & $\chi(-1) \chi^{6}(2) J_{\chi}(2,2)$ \\
\hline$J_{\chi}(6, n)$ & $\chi^{6}(2) J_{\chi}(3,1)$ & -1 \\
\hline \multirow[t]{2}{*}{$J_{\chi}(7, n)$} & $-\chi(-1)$ & $\chi^{2}(2) J_{\bar{\chi}}(3,1)$ \\
\hline & $n=3$ & $n=4$ \\
\hline$J_{\chi}(0, n)$ & -1 & -1 \\
\hline$J_{\chi}(1, n)$ & $J_{\chi}(3,1)$ & $\chi(-1) J_{\chi}(3,1)$ \\
\hline$J_{\chi}(2, n)$ & $\chi^{2}(2) J_{\chi}(3,1)$ & $J_{\chi}(2,2)$ \\
\hline$J_{\chi}(3, n)$ & $\chi(-1) \chi^{2}(2) J_{\chi}(3,1)$ & $\chi(-1) J_{x}(3,1)$ \\
\hline$J_{\chi}(4, n)$ & $\chi(-1) J_{\chi}(3,1)$ & -1 \\
\hline$J_{\chi}(5, n)$ & $-\chi(-1)$ & $\chi(-1) J_{\bar{\chi}}(3,1)$ \\
\hline$J_{\chi}(6, n)$ & $\chi(-1) \chi^{2}(2) J_{\bar{\chi}}(2,2)$ & $J_{\bar{\chi}}(2,2)$ \\
\hline$J_{\chi}(7, n)$ & $\chi^{2}(2) J_{\bar{\chi}}(2,2)$ & $\chi(-1) J_{\bar{\chi}}(3,1)$ \\
\hline
\end{tabular}

The entries in the table follow from one or more of the formulas $(2.8), \cdots,(2.14)$. For example, the formula for $J_{\chi}(2,1)$ is obtained from (2.14) upon replacing $\alpha$ by $\beta$ and then applying (2.10) and (2.13). Again, if in (2.14) $\alpha$ is replaced by $\beta^{3}$ and both members of the resulting equation are multiplied by $\tau(\beta) / \tau\left(\beta^{4}\right) \tau\left(\beta^{7}\right)$, then the formula for $J_{\chi}(1,1)$ follows. The other entries are similarly established.

By means of Lemma 4 with $e=8$ we obtain the following results:

$$
\begin{aligned}
S_{1}(g)= & \frac{1}{4}\left\{1+\chi^{6}(2) \psi^{2}(2) J_{\chi}(3,1) J_{\Psi}(3,1)\right. \\
& +\chi(-1) \psi(-1) \chi^{6}(2) \psi^{2}(2) J_{\chi}(2,2) J_{\bar{\psi}}(2,2) \\
& \left.+\chi(-1) \psi(-1) J_{\chi}(3,1) J_{\bar{\psi}}(3,1)\right\},
\end{aligned}
$$




$$
\begin{aligned}
S_{2}(g)= & \frac{1}{8}\left\{2+2 J_{\chi}(2,2) J_{\bar{\psi}}(2,2)+2 \chi^{6}(2) \psi^{2}(2) J_{\chi}(2,2) J_{\bar{\psi}}(2,2)\right. \\
& \left.+\chi(-1) \psi(-1) \chi^{2}(2) \psi^{6}(2)\left(J_{\chi}(3,1) J_{\bar{\psi}}(3,1)+J_{\bar{\chi}}(3,1) J_{\psi}(3,1)\right)\right\},
\end{aligned}
$$

$S_{3}(g)=\frac{1}{4}\left\{1+\left(\chi(-1) \psi(-1)+\chi^{2}(2) \psi^{6}(2)\right) J_{\chi}(3,1) J_{\Psi}(3,1)\right.$

$$
\left.+\chi(-1) \psi(-1) \chi^{2}(2) \psi^{6}(2) J_{\bar{\chi}}(2,2) J_{\psi}(2,2)\right\},
$$

$$
\begin{aligned}
S_{4}(g)=\frac{1}{8}\left\{2+2\left(J_{\chi}(3,1) J_{\bar{\psi}}(3,1)+\right.\right. & J_{\bar{\chi}}(3,1) J_{\psi}(3,1) \\
& \left.+\left(J_{\chi}(2,2) J_{\bar{\psi}}(2,2)+J_{\bar{\chi}}(2,2) J_{\psi}(2,2)\right)\right\} .
\end{aligned}
$$

We now apply Theorem 1 with $e=8$. The result of substituting (7.1), (7.2), (7.3), (7.4) into (2.21) may be summarized as follows. Let $M=((p-2)(q-2)-1) / 8$. Then

$$
\begin{aligned}
64 N_{8}(g)= & 8 M+8 \eta+14+\delta\left(J_{\chi}(3,1) J_{\bar{\psi}}(3,1)+J_{\bar{\chi}}(3,1) J_{\psi}(3,1)\right) \\
& +\varepsilon\left(J_{\chi}(2,2) J_{\bar{\psi}}(2,2)+J_{\bar{\chi}}(2,2) J_{\psi}(2,2)\right),
\end{aligned}
$$

where

$$
\delta= \begin{cases}-2+\chi^{2}(2) \psi^{6}(2)+\chi^{6}(2) \psi^{2}(2) & \left(f f^{\prime} \text { even }\right) \\ 6+3 \chi^{2}(2) \psi^{6}(2)+3 \chi^{6}(2) \psi^{2}(2) & \left(f f^{\prime} \text { odd }\right)\end{cases}
$$

and

$$
\varepsilon= \begin{cases}3-2 \chi^{6}(2) \psi^{2}(2) & \left(f f^{\prime} \text { even }\right) \\ 3+6 \chi^{6}(2) \psi^{2}(2) & \left(f f^{\prime} \text { odd }\right)\end{cases}
$$

In view of (5.5) we put

$$
J_{\chi}(2,2)=-x+2 \beta^{2} y, \quad J_{\psi}(2,2)=-X+2 \beta^{2} Y,
$$

where

$$
p=x^{2}+4 y^{2}, \quad q=X^{2}+4 Y^{2} \quad(x \equiv X \equiv 1(\bmod 4)) .
$$

Following Dickson [1, p. 411], we also put

$$
J_{\chi}(3,1)=-a+b\left(\beta+\beta^{3}\right), \quad J_{\psi}(3,1)=-A+B\left(\beta+\beta^{3}\right),
$$

where

$$
p=a^{2}+2 b^{2}, \quad q=A^{2}+2 B^{2} .
$$

Dickson [1, pp. 411-413] proved that $a \equiv \pm 1(\bmod 4)$ according as $f$ is even or odd; similarly $A \equiv \pm 1(\bmod 4)$ according as $f^{\prime}$ is even or odd. On the other hand, the signs of $b, B$ are ambiguous and depend on the selection of $g$.

Since $(2 \mid p)=1,(2 \mid q)=1$ it follows that $\chi^{2}(2)= \pm 1, \psi^{2}(2)= \pm 1$. Hence the numbers $\delta, \varepsilon$ in (7.6), (7.7) are real. Indeed, if $f f^{\prime}$ is even, then $\delta=0$ or -4 and 
$\varepsilon=1$ or 5 according as $\chi^{2}(2) \psi^{6}(2)= \pm 1$; if $f f^{\prime}$ is odd, then $\delta=12$ or 0 and $\varepsilon=9$ or -3 according as $\psi^{2}(2) \psi^{6}(2)= \pm 1$. Substituting from (7.8), (7.10) into (7.5) we obtain the following theorem.

THEOREM 5. When $e=8$ the number $N_{8}(g)$ of solutions of (2.2) satisfies

$$
64 N_{8}(g)=\left\{\begin{array}{c}
8 M+14+2(x X+4 y Y) \\
\quad\left(f f^{\prime} \text { even, } \chi^{2}(2) \psi^{6}(2)=1\right), \\
8 M+14-8(a A+2 b B)+10(x X+4 y Y) \\
\left(f f^{\prime} \text { even, } \chi^{2}(2) \psi^{6}(2)=-1\right), \\
8 M+22+24(a A+2 b B)+18(x X+4 y Y) \\
\left(f f^{\prime} \text { odd }, \chi^{2}(2) \psi^{6}(2)=1\right), \\
8 M+22-6(x X+4 y Y) \\
\left(f f^{\prime} \text { odd }, \chi^{2}(2) \psi^{6}(2)=-1\right) .
\end{array}\right.
$$

Because $y, Y, b, B$ are ambiguous, formula (7.12) is valid only for some unspecified choices of $g$. More explicit expressions may be derived if we make use of the classification of primitive roots given in Lemma 6 with $e=8$. However, we shall not take the space to deduce the resulting formulas.

8. The modulus pqr. Let $g$ be a common primitive root of distinct odd primes $p, q, r$. The exponent $d$ to which $g$ belongs modulo $p q r$ is the least common multiple of $p-1, q-1, r-1$. Making the assumption

$$
(p-1, q-1)=(q-1, r-1)=(r-1, p-1)=2,
$$

we find that $d=(p-1)(q-1)(r-1) / 4$. In this section we derive explicit expressions for the number $N(g)$ of solutions of the congruence

$$
g^{s}+1 \equiv g^{t}(\bmod p q r)
$$

where $s$ and $t$ are each selected from $0,1, \cdots, d-1$. The method is essentially that of $\S 2$.

We define three Legendre characters as follows: $\chi(n)=(n \mid p), \psi(n)=(n \mid q)$, $\lambda(n)=(n \mid r)$. The argument used to prove Lemma 1 yields the following criterion. Let $s$ be a fixed integer such that $0 \leqq s \leqq d-1$ and $\left(g^{s}+1, p q r\right)=1$. Then there corresponds an integer $t$ such that $0 \leqq t \leqq d-1$ and $g^{s}+1 \equiv g^{t}(\bmod p q r)$ if and only if $\chi\left(g^{s}+1\right)=\psi\left(g^{s}+1\right)=\lambda\left(g^{s}+1\right)$.

Let $T(g)$ denote the number of values of $s$ such that $0 \leqq s \leqq d-1$ and $\left(g^{s}+1, p q r\right)=1$. It is clear that the number $N(g)$ of solutions of (8.2) is related to the sum 


$$
\begin{aligned}
S(g)= & \sum_{s=0}^{d-1}\left\{1+\chi^{2}\left(g^{s}+1\right) \psi\left(g^{s}+1\right) \lambda\left(g^{s}+1\right)\right. \\
& \left.\quad+\chi\left(g^{s}+1\right) \psi^{2}\left(g^{s}+1\right) \lambda\left(g^{s}+1\right)+\chi\left(g^{s}+1\right) \psi\left(g^{s}+1\right) \lambda^{2}\left(g^{s}+1\right)\right\}
\end{aligned}
$$

by means of the equation

$$
S(g)=4 N(g)+T(g)
$$

We proceed to compute $T(g)$. For an integer $v$ let $N_{v}$ denote the number of values of $s(s=0,1, \cdots, d-1)$ for which $N=N(s)=1+g^{s}$ is divisible by $v$. It follows from a familiar combinatorial principle that $T(g)=N_{p}+N_{q}+N_{r}-N_{p q}-N_{p r}-N_{q r}+N_{p q r}$. We first evaluate $N_{p q r}$. Put $p-1=2 f, q-1=2 f^{\prime}, r-1=2 f^{\prime \prime}$. By (8.1) at most one of $f, f^{\prime}, f^{\prime \prime}$ is even. If $f f^{\prime} f^{\prime \prime}$ is odd, then $-1 \equiv g^{d / 2}(\bmod p q r)$. But if $f f^{\prime} f^{\prime \prime}$ is even, then there is no value of $s$ such that $-1 \equiv g^{s}(\bmod p q r)$, where $0 \leqq s \leqq d-1$. Hence $N_{p q r}$ is equal to 0 or 1 according as $f f^{\prime} f^{\prime \prime}$ is even or odd. We next determine $N_{p q}$. When $f f^{\prime}$ is even the congruence $g^{s}+1 \equiv 0(\bmod p q), 0 \leqq s \leqq d-1$, is not solvable. But when $f f^{\prime}$ is odd this congruence is solvable if and only if $s$ is an odd multiple of $(p-1)(q-1) / 4$. There are $(r-1) / 2$ such values of $s$ between 0 and $d-1$. Thus $N_{p q}=0$ or $f^{\prime \prime}$ according as $f f^{\prime}$ is even or odd; similarly, $N_{p r}=0$ or $f^{\prime}$ according as $f f^{\prime \prime}$ is even or odd, and $N_{q r}=0$ or $f$ according as $f^{\prime} f^{\prime \prime}$ is even or odd. Finally, we evaluate $N_{p}$. As $s$ ranges from 0 to $d-1$, the least positive remainders of $g^{s}$ modulo $p$ range $f^{\prime} f^{\prime \prime}$ times over each of the integers between 1 and $p-1$. Hence $N_{p}=f^{\prime} f^{\prime \prime}$. Similarly, $N_{q}=f f^{\prime \prime}$ and $N_{r}=f f^{\prime}$. From the results of this paragraph we obtain the following relations.

$$
T(g)= \begin{cases}f f^{\prime}+f f^{\prime \prime}+f^{\prime} f^{\prime \prime}-f & (f \text { even }), \\ f f^{\prime}+f f^{\prime \prime}+f^{\prime} f^{\prime \prime}-f^{\prime} & \left(f^{\prime} \text { even }\right), \\ f f^{\prime}+f f^{\prime \prime}+f^{\prime} f^{\prime \prime}-f^{\prime \prime} & \left(f^{\prime \prime} \text { even }\right), \\ f f^{\prime}+f f^{\prime \prime}+f^{\prime} f^{\prime \prime}-f-f^{\prime}-f^{\prime \prime}-1 & \left(f f^{\prime} f^{\prime \prime} \text { odd }\right) .\end{cases}
$$

To compute $S(g)$ we write (8.3) in the form $S(g)=d+S_{1}(g)+S_{2}(g)+S_{3}(g)$, where

$$
\begin{aligned}
& S_{1}(g)=\sum_{s=0}^{d-1} \chi^{2}\left(g^{s}+1\right) \psi\left(g^{s}+1\right) \lambda\left(g^{s}+1\right), \\
& S_{2}(g)=\sum_{s=0}^{d-1} \chi\left(g^{s}+1\right) \psi^{2}\left(g^{s}+1\right) \lambda\left(g^{s}+1\right), \\
& S_{3}(g)=\sum_{s=0}^{d-1} \chi\left(g^{s}+1\right) \psi\left(g^{s}+1\right) \lambda^{2}\left(g^{s}+1\right) .
\end{aligned}
$$

Consider the first equation in (8.6). Let $u, v, w$ run through the quadratic residues of $p, q, r$, respectively. Then this equation may be transformed into 


$$
\begin{aligned}
S_{1}(g) & =\sum_{k=0}^{1} \sum_{u, v, w} \chi^{2}\left(g^{k} u+1\right) \psi\left(g^{k} v+1\right) \lambda\left(g^{k} w+1\right) \\
& =\frac{1}{8} \sum_{k=0}^{1} \sum_{a=1}^{p-1} \chi^{2}\left(g^{k} a^{2}+1\right) \sum_{b=1}^{p-1} \psi\left(g^{k} b^{2}+1\right) \sum_{c=1}^{p-1} \lambda\left(g^{k} c^{2}+1\right) .
\end{aligned}
$$

Employing the easily established formulas

$$
\sum_{a=1}^{p-1} \chi\left(n a^{2}+1\right)=-1-\chi(n), \quad \sum_{a=1}^{p-1} \chi^{2}\left(n a^{2}+1\right)=p-2-\chi(-n),
$$

we find that

$$
S_{1}(g)=\frac{1}{8} \sum_{k=0}^{1}\left(p-2-\chi\left(-g^{k}\right)\right)\left(-1-\psi\left(g^{k}\right)\right)\left(-1-\lambda\left(g^{k}\right)\right)
$$

This reduces readily to the first equation of

$$
\begin{aligned}
& S_{1}(g)=\frac{1}{2}(p-2-\chi(-1)), \\
& S_{2}(g)=\frac{1}{2}(q-2-\psi(-1)), \\
& S_{3}(g)=\frac{1}{2}(r-2-\lambda(-1)) .
\end{aligned}
$$

Because of symmetry the last two equations of (8.6) are transformed into the last two equations of (8.7).

The following theorem now follows from (8.4), (8.5) and (8.7).

THEOREM 6. The number $N(g)$ of solutions of (8.2) is given by

$$
16 N(g)= \begin{cases}(p-2)(q-2)(r-2)+3 p+q+r-8 & (f \text { even }), \\ (p-2)(q-2)(r-2)+p+3 q+r-8 & \left(f^{\prime} \text { even }\right), \\ (p-2)(q-2)(r-2)+p+q+3 r-8 & \left(f^{\prime \prime} \text { even }\right), \\ (p-2)(q-2)(r-2)+3 p+3 q+3 r-12 & \left(f f^{\prime} f^{\prime \prime} \text { odd }\right)\end{cases}
$$

It is clear from (8.8) that the value of $N(g)$ is independent of the choice of $g$.

\section{REFERENCES}

1. L. E. Dickson, Cyclotomy, higher congruences, and Waring's problem, Amer. J. Math. 57 (1935), 391-424.

2. W. J. LeVeque, Topics in number theory, Vol. 1, Addison-Wesley, Reading, Mass., 1956.

3. A. L. Whiteman, A family of difference sets, Illinois J. Math. 6 (1962), 107-121.

DUKE UNIVERSTTY,

DURHAM, North Carolina

UNIVERSITY OF SOUTHERn CALIFornia,

Los Angeles, California 